\title{
Persistent atomic spin squeezing at the Heisenberg limit
}

\author{
Ling-Na Wu, ${ }^{1}$ Meng Khoon Tey, ${ }^{1,2}$ and L. You ${ }^{1,2, *}$ \\ ${ }^{1}$ State Key Laboratory of Low Dimensional Quantum Physics, \\ Department of Physics, Tsinghua University, Beijing 100084, China \\ ${ }^{2}$ Collaborative Innovation Center of Quantum Matter, Beijing 100084, China
}

(Dated: August 29, 2018)

\begin{abstract}
Two well-known mechanisms, one-axis twisting (OAT) and two-axis counter twisting (TACT), generate spin squeezed states dynamically. The latter provides better spin squeezing (SS), but has not been demonstrated as the form of its interaction does not occur naturally in known physical systems. Several proposals for realizing effective TACT transformed from OAT require stringent experimental conditions, in order to overcome the resulting non-stationary (oscillating) SS and continuously varying mean spin directions. This work presents a simple scheme that solves both problems by freezing SS at an optimal point and realizing effectively persistent SS by inhibiting further squeezing dynamics. Explicit procedures are outlined for persistent SS of the TACT limit. Protocols based on our scheme favorably relax experimental demands, which significantly brighten the prospects for realizing TACT.
\end{abstract}

Recent successes in atomic spin squeezing (SS) have significantly raised the prospects for its application to high precision measurement [1-13] and to entanglement detection [14-19]. Squeezed spin state (SSS) [1, 20, 21] is a state of many spin (pseudo-spin) $1 / 2$ particles with exchange symmetry, whose uncertainty in one collective spin component perpendicular to the mean spin direction is smaller than the classical limit set by coherent spin state (CSS), where all spins are identically aligned up in the same direction. Kitagawa and Ueda [20] proposed two well-known mechanisms for generating SS with one-axis twisting (OAT) and two-axis counter twisting (TACT) interactions, which are described respectively by the Hamiltonians $H_{\mathrm{OAT}}=\chi J_{z}^{2}$ and $H_{\mathrm{TACT}}=$ $\chi\left(J_{z}^{2}-J_{y}^{2}\right)$. Here $J_{i}=\sum_{k} \sigma_{i}^{(k)} / 2(i=x, y, z)$ denote the collective spin components, where $\sigma_{i}^{(k)}$ are the Pauli matrices for the $\mathrm{k}$-th spin $1 / 2$ particle, and $\chi$ denotes the identical strength of coupling between two spins. The squeezing parameter $\xi^{2}=\left(\Delta J_{\perp}\right)_{\min }^{2} /\left(\Delta J_{\perp}\right)_{\mathrm{CSS}}^{2}$ quantifies the degree of SS in terms of the minimum of the fluctuation $\left(\Delta J_{\perp}\right)^{2}=\left\langle J_{\perp}^{2}\right\rangle-\left\langle J_{\perp}\right\rangle^{2}$ for the collective spin component perpendicular to the mean spin $\langle\vec{J}\rangle=\left(\left\langle J_{x}\right\rangle,\left\langle J_{y}\right\rangle,\left\langle J_{z}\right\rangle\right)$ relative to $\left(\Delta J_{\perp}\right)_{\mathrm{CSS}}^{2}=N / 4$ for a CSS with $N$ the number of particles.

The theoretical limits of the squeezing parameters for OAT and TACT scale as $\propto N^{-2 / 3}$ and $\propto N^{-1}$, respectively [20]. Despite its better scaling capable of approaching within a few times of the Heisenberg limit $(1 / N)$, TACT is yet to be demonstrated as its form of interaction does not occur naturally in most systems of interest for studying SS. OAT, on the other hand, has been implemented in many systems [4, 5, 22-25]. Many theoretical studies have been carried out to realize TACT interactions [26-33]. Two promising proposals realize TACT by coherent manipulation of OAT [26, 27]. Specifically, in Ref. [26], an alternating $\pi / 2$ pulse train periodically

*lyou@mail.tsinghua.edu.cn switches the OAT axis; and in Ref. [27], a periodically modulated drive is applied to continuously modify the direction of atomic spin. Although TACT SS is realized in both cases at integer periods of modulation, the amount of SS oscillates in time with an amplitude determined by the degree of how well the approximation conditions adopted $[26,27]$ are satisfied. For instance, in the formal proposal [26], 1000 or more pulses are required for a condensate with $N=1250$ atoms in order to reach a squeezing parameter $\left(\xi^{2}\right)$ within twice the limit of TACT. Without impeccable precisions, however, errors from repeated pulses will accumulate to spoil the intended dynamic spin control. In the latter proposal [27], modulation frequencies as high as $10^{5}$ times the coupling strength $\chi$ and Rabi frequencies of the same order of magnitude are required at the same $N$. Additionally the continuously nutating mean spin direction from modulated drive complicates its detection. Therefore, schemes capable of effectively suppressing SS $\left(\xi^{2}\right)$ oscillation amplitude and tracking the varying mean spin direction would significantly establish the proposals' feasibilities.

This paper presents an idea which solves both aforementioned challenges in the two proposals [26, 27]. Its application freezes squeezing dynamics at the theoretically determined optimal point, essentially achieves persistent TACT squeezing. The following discussion starts with a description of our basic idea. Explicit operation protocols are then provided for the two afore-mentioned proposals [26, 27], which lead respectively to more than one order of magnitude reduction in the required number of pulses and five times reduction in the modulation (and Rabi) frequency.

We note that a many particle state with a sharp distribution around a system eigenstate is less sensitive to dynamic evolution [34]. For example, we consider the OAT model $H_{\mathrm{OAT}}=\chi J_{z}^{2}$, whose eigenstates are $\{|j, m\rangle\}$, satisfying $J_{z}|j, m\rangle=m|j, m\rangle$, with $m=-j,-j+1, \ldots, j$. The eigenstate $|j, m\rangle$ acquires a phase $\chi t m^{2}$ after time $t$. A state with a wide distribution of $m$ phase diffuses [35] quickly. A state with a narrow distribution around a 
particular eigenstate $|j, k\rangle$, however, diffuses slowly and essentially acquires a global phase factor $\chi t k^{2}$. When $k \sim 0$, its temporal evolution becomes effectively frozen.

The above discussion illustrates the basics of a robust storage scheme for SS [34] in the model of OAT with a constant drive [36], whereby the spin distribution is in continuous rotation around the mean spin direction while undergoing SS. The drive is turned off at precisely the moment when optimal SS lies along the $z$-axis [34]. The corresponding SSS becomes insensitive to subsequent dynamical evolution due to its squeezed distribution $(k \sim 0)$ along the $z$-axis. Instead of waiting passively for the optimal moment, our idea is on the active side. We propose to rotate the maximally squeezed direction of a SSS to along the $z$-axis instantaneously when optimal squeezing occurs, which freezes optimal SS and keeps it persistent. In order to precisely carry out the rotation, we need to know the optimal squeezing direction. Likewise, to reach optimal SS, an accurate knowledge of the optimal squeezing time is required. Fortunately, these information are known for both OAT and TACT models, e.g., the optimal squeezing time for OAT and TACT are $\chi t_{\mathrm{opt}}^{(\mathrm{OAT})} \simeq 6^{1 / 6} N^{-2 / 3}[20]$ and $\chi t_{\mathrm{opt}}^{(\mathrm{TACT})} \simeq \ln (4 N) /(2 N)$ [26], respectively. Although both depend on atom number $N$, this dependence is very weak for the squeezing parameter $\xi^{2}$ in the vicinity of the maximal squeezing, which greatly relaxes the required precision of temporal controls. The optimal squeezing direction is fixed in the TACT model, independent of $N$ or other parameters, which is an advantage over the OAT model.

The following discussions detail the operation protocols for our scheme when applied to the two aforementioned proposals [26, 27]. First, consider the repeated pulse proposal [26], where a $\pi / 2$ pulse train periodically switches the OAT axis [Fig. 1(a)]. Each period lasts for $t_{c}=3 \delta t$ and is composed of two parts: an evolution of $2 \delta t$ (red shadow) governed by OAT Hamiltonian $H_{1}=\chi J_{x}^{2}$, followed by OAT $H_{2}=H_{\mathrm{OAT}}$ for the remaining $\delta t$ (blue shadow). $H_{1}$ is transformed from $H_{\mathrm{OAT}}$ by a pair of $\pi / 2$ pulses (gray rectangles) applied along the $\pm y$ axis. Although they do not commute, i.e. $\left[H_{1}, H_{2}\right] \neq 0$, provided $2 \chi \delta t N \ll 1$, we can neglect higher order terms in $\delta t$ and end up with an effective TACT Hamiltonian $H_{\text {eff }}=\left(2 H_{1}+H_{2}\right) / 3=\chi\left(J_{x}^{2}-J_{y}^{2}\right) / 3$ apart from a constant $J^{2}=j(j+1)$. As shown in Fig. 1(b), SS from the actual pulse sequence reaches the TACT limit (black dashed line) at specific times, accompanied with oscillations from the higher order terms in $\delta t$. The accuracy of this approximation can be improved by reducing $\delta t$, which shortens $t_{c}$ and inhibits oscillation amplitudes. However, this is not always a winning strategy as the optimal squeezing time $t_{\text {opt }}$ is fixed by $N$. A smaller $t_{c}$ indicates a larger $N_{c}$, which denotes the nearest integer number of pulse pairs needed to reach optimal SS. It was found earlier that more than $N_{c}=1000$ pairs of pulses are needed to reach the effective TACT at $N=1250$ [26]. Controlling such a large number of identical pulses to the required accuracies is a serious experimental challenge.

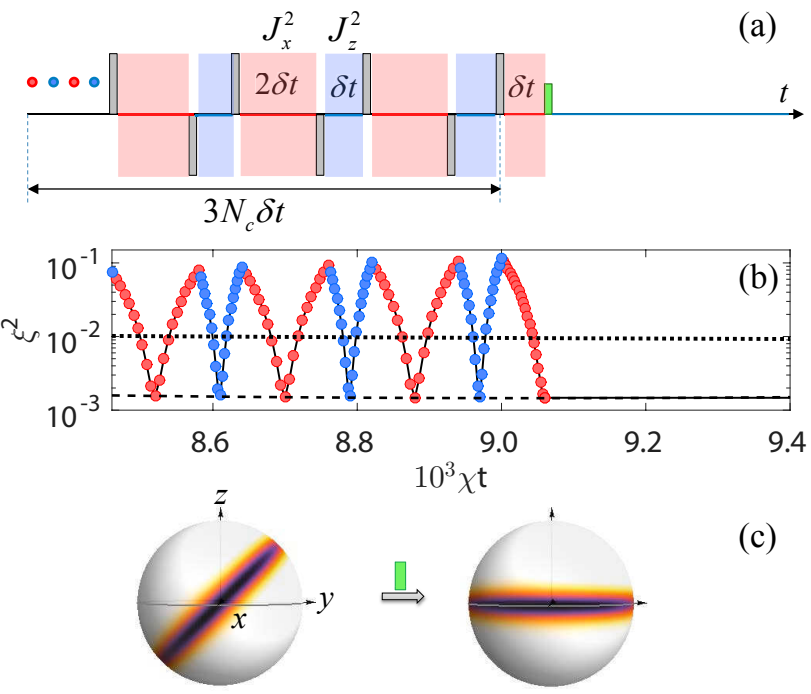

FIG. 1. (Color online) (a) An illustration of the repeated pulse proposal [26], where each period lasts for $t_{c}=3 \delta t$ and is composed of a $2 \delta t$ section (red shadow) governed by $H_{1}=\chi J_{x}^{2}$ and a $\delta t$ section (blue shadow) governed by $H_{2}=\chi J_{z}^{2} \cdot H_{1}$ is transformed from $H_{2}$ by a pair of $\pi / 2$ pulses (gray rectangles) applied along the $\pm y$-axis. The green rectangle represents the rotation pulse proposed to freeze spin evolution. (b) The evolution of squeezing parameter $\xi^{2}$ as a function of time. Red (blue) disks denote the first (second) part of duration $2 \delta t(\delta t)$. Black solid line represents the result upon implementation of the present scheme. For all figures in this work, black dashed line and black dotted line refers to the limits of TACT and OAT models, respectively. (c) A Bloch sphere illustration of the rotation operation conducted to freeze SS. The quasi-probability distribution for a state $|\psi(t)\rangle$ is $|\langle\theta, \varphi \mid \psi(t)\rangle|^{2}$, where $|\theta, \varphi\rangle$ denotes CSS pointing along $(\theta, \varphi)$ direction. The initial state used for our scheme is $|j, j\rangle$, while the limits of OAT and TACT in all figures in this work are obtained with their appropriate CSS as initial states. Likewise, in this and all other figures shown later, $N=1250$ is used, except for illustrations with Bloch spheres where $N=100$ is used as in (c) for enhanced details of the quasi-probability distribution, and the number of periods $N_{c}=50$.

The good news lies at the fact that even for $N_{c}=50$, despite its oscillations, $\xi^{2}$ already touches the TACT limit at approximately $\left(N_{c} \pm n\right) t_{c}+\delta t$ and $\left(N_{c} \pm n\right) t_{c}+$ $2.5 \delta t$ for integers $n=0,1,2, \cdots$ and $N_{c} t_{c} \simeq t_{\text {opt }}=$ $3 t_{\text {opt }}^{\text {(TACT) }}$ [Fig. 1(b)]. Our protocol calls for the rotation of the optimal squeezed direction to along the $z$-axis with a short pulse [green rectangles in Fig. 1(a) and 1(c)], at the appropriate moments when optimal SS is reached. This freezes the optimal SS [black solid line in Fig. 1(b)], which nearly overlaps with TACT limit. The squeezing axis at maximum squeezing lies at an angle $\pi / 4$ to the $z$-axis in the $y$-z plane [26], which can be rotated to the $z$-axis by a $\pi / 4$ pulse along the $-x$-axis, as shown in Fig. 1 (c). Given $\chi \sim(2 \pi) 0.063 \mathrm{~Hz}$ as from recent experiment [4] and $\chi t_{\text {opt }} \sim 0.01$ for $N=1250$ [Fig. 1(b)], we have $N_{c} t_{c} \sim 25 \mathrm{~ms}$. At $N_{c}=50$, a single pulse is limited to a duration $\leq 10 \mu \mathrm{s}$ as $t_{c} \sim 500 \mu \mathrm{s}$ or $\delta t \sim 170 \mu \mathrm{s}$, which is 


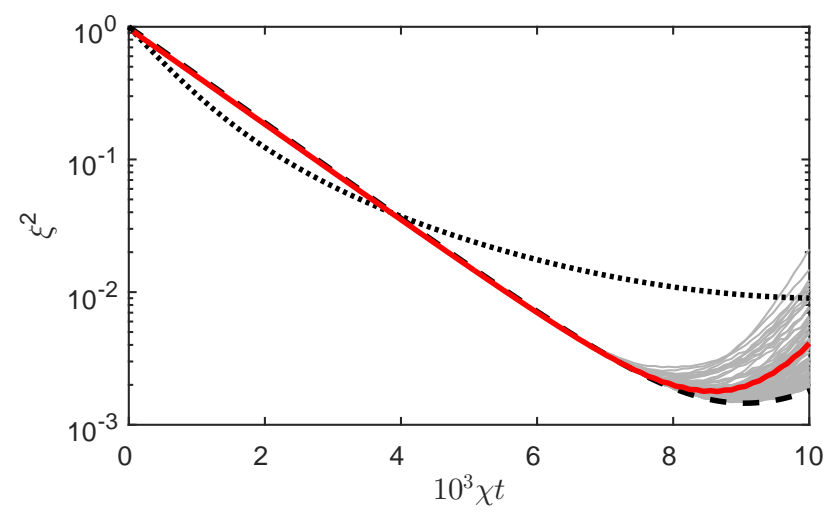

FIG. 2. (Color online) The evolution of squeezing parameter $\xi^{2}$ for the repeated pulse proposal in the presence of added linear noise to the pulse area according to a model described in the main text. The grey lines show $\xi^{2}$ from 100 independent realizations, and the thick red line denotes their average. For easy viewing of the oscillating $\mathrm{SS}$, only data points at $n t_{c}+\delta t$ and $n t_{c}+2.5 \delta t$ for $n=0,1,2, \cdots$, are shown.

now feasible in contrast to the 1000 pulse pairs originally required. This drastic reduction of $N_{c}$ also makes the proposal more robust to a fluctuating pulse area. Figure 2 displays squeezing parameters $\xi^{2}$ from 100 independent simulations for a fluctuating pulse area proportionally scaled to $1+r \eta$, with $\eta=0.1 \%$ and $r \in[-0.5,0.5]$ a uniformly distributed random number. Such conditions are achievable experimentally, and our simulations show that the quality of SS remains very good. In particular, we note that in the broad temporal domain before the optimal SS is reached, $\xi^{2}$ is found to track the limit of TACT, essentially unaffected by the fluctuating noise and at a level significantly below the limit of OAT.

We now apply our protocol to the modulated drive proposal [27], where a continuously modulated drive $\Omega(t)=\Omega_{0} \cos (\omega t+\varphi)$ augments the OAT model to give

$$
H(t)=H_{\mathrm{OAT}}+\Omega(t) J_{y} .
$$

In the high-frequency limit $\omega \gg N \chi$, this Hamiltonian at $\varphi=0$ is well approximated by

$$
H_{\mathrm{appx}}=\chi\left[\alpha_{0} J_{z}^{2}+\left(1-\alpha_{0}\right) J_{x}^{2}\right],
$$

with $\alpha_{0}=\frac{1}{2}\left[1+J_{0}\left(2 \Omega_{0} / \omega\right)\right]$, where $J_{\nu}($.$) denotes the \nu$ th order Bessel function, and is bounded within $(-0.5,1]$. At $\Omega_{0} / \omega=0.9057, \alpha_{0}=2 / 3$, Hamiltonian (2) reduces to

$$
H_{\mathrm{eff}}=\frac{\chi}{3}\left(2 J_{z}^{2}+J_{x}^{2}\right)=\frac{\chi}{3}\left(J^{2}+J_{z}^{2}-J_{y}^{2}\right),
$$

which is formally equivalent to the TACT model, except for a constant $J^{2}=j(j+1)$ term. Heisenberg limited SS can be achieved dynamically starting from a coherent state $|j, j\rangle_{x}=\exp \left(-i \pi J_{y} / 2\right)|j, j\rangle$ with all spins pointing to the $x$-direction.

In fact, this effective TACT remains applicable even for an arbitrary phase $\varphi \neq 0$ except for an extra unitary transformation (see appendix)

$$
H_{\mathrm{eff}}^{\prime}=R_{y}(\varphi) H_{\mathrm{eff}} R_{y}(-\varphi),
$$

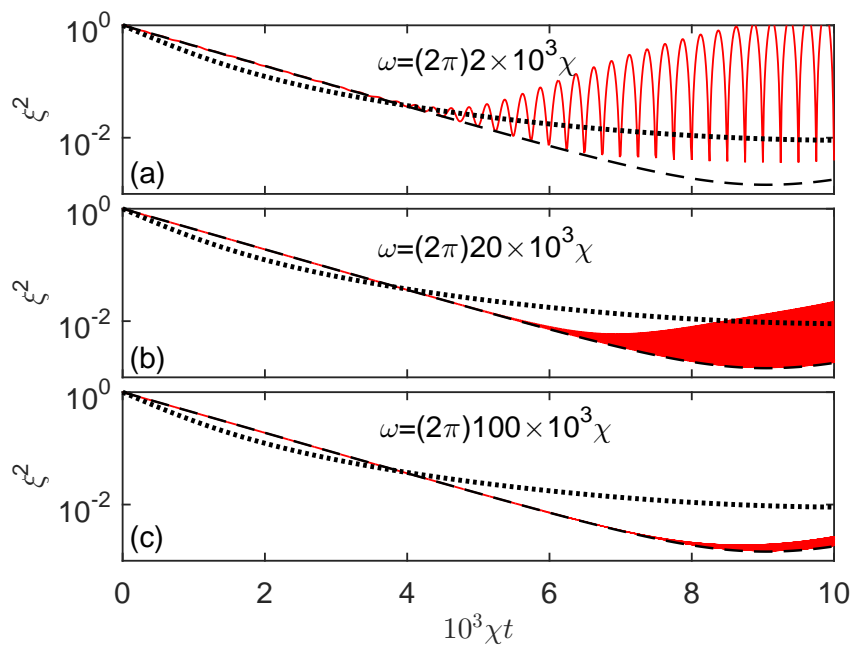

FIG. 3. (Color online) Squeezing parameter $\xi^{2}$ from the actual dynamics of the Hamiltonian (1) with $\varphi=-\pi / 2$ (red solid lines) at $\omega=(2 \pi) 2 \times 10^{3} \chi(\mathrm{a}),(2 \pi) 2 \times 10^{4} \chi(\mathrm{b}),(2 \pi) 10^{5} \chi(\mathrm{c})$ for a fixed ratio of $\Omega_{0} / \omega=0.9057$. The initial state used for the modulated drive proposal is $\exp \left(i \Omega_{0} J_{y} / \omega\right)|j, j\rangle_{x}$.

where $R_{y}(\varphi)=\exp \left(-i \frac{\Omega_{0}}{\omega} \sin \varphi J_{y}\right)$ denotes a rotation around the $y$-axis by an angle $\left(\Omega_{0} / \omega\right) \sin \varphi$. SS at Heisenberg limit is again realized if a rotated CSS $R_{y}(\varphi)|j, j\rangle_{x}$ is used as the initial state.

Figure 3 displays $\xi^{2}$ from the dynamics of the modulated drive proposal (red solid lines). Fixing $\Omega_{0}=$ $0.9057 \omega, \xi^{2}$ approaches and eventually overlaps completely with results from the effective dynamics of $H_{\text {eff }}$ (3) (black dashed lines) when the modulation frequency $\omega$ increases. To suppress oscillation amplitude to within $50 \%$ of the TACT limit, the high frequency approximation requires $\omega \geq(2 \pi) 10^{5} \chi$ at $N=1250$, which implies an equally large Rabi frequency $\Omega_{0} \sim \omega$ of about $(2 \pi) 40 \mathrm{kHz}$ using $\chi=(2 \pi) 0.063 \mathrm{~Hz}$. These are challenging conditions when $\chi$ and $N$ are both large. Additionally, SS in this case is accompanied by a continuous nutation of the mean spin direction, which is in contrast to the TACT model, where both the mean spin direction and the maximal squeezing direction are fixed [20].

The difficulties associated with detecting an oscillating SS parameter and a nutating mean spin direction can again be solved altogether if we apply our idea to freeze SS at the optimal [Fig. 4(a)]. Figure 4(b) and 4 (c) respectively show SS parameter $\xi^{2}$ and the modulation drive for the time window marked by the blue dot-dashed rectangle in Fig. 4(a). The coupling $\Omega(t) J_{y}$ from the modulated drive in Fig. 4(c) continuously nutates the state around the $y$-axis, causing its mean spin $\langle\vec{J}\rangle$ to oscillate between the northern and southern hemispheres [Fig. $4(\mathrm{~d})] . \quad\langle\vec{J}\rangle$ reaches its highest or lowest excursion [(i),(iii)] when $\Omega(t)=0$ at the moments of optimal SS [Fig. 4(b)]. The rotation angle of the solstice relative to the equatorial plane is estimated to be $\int_{0}^{T / 4} \Omega(t) d t=\Omega_{0} / \omega$ with $T=2 \pi / \omega$ the period, if we 


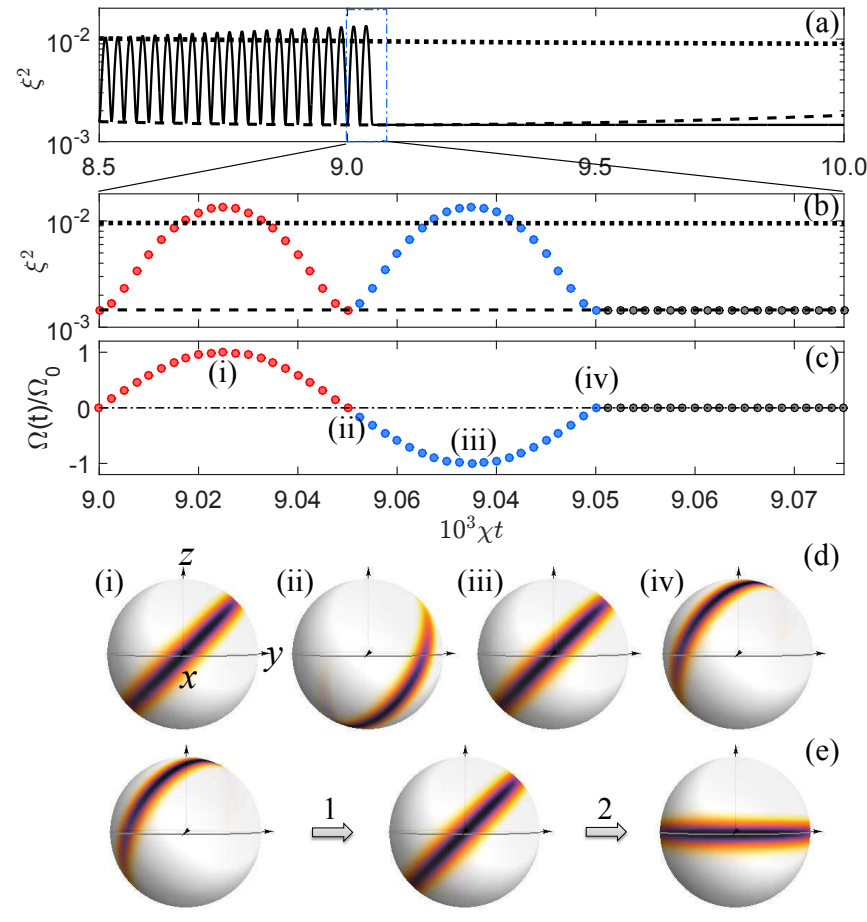

FIG. 4. (Color online) Freezing of the SS governed by Hamiltonian (1) with $\varphi=-\pi / 2$. (a) Squeezing parameter $\xi^{2}$ as a function of time. The black solid line denotes the result of the modulated drive proposal aided by our protocol. (b)-(c) correspond to the zoomed in region marked by the blue dot-dashed rectangle in (a), with (b) displaying $\xi^{2}$ and (c) showing the scaled drive $\Omega(t) / \Omega_{0}$; (d) displays the time-dependent quasiprobability distribution $|\langle\theta, \varphi \mid \psi(t)\rangle|^{2}$ at moments marked (i)(iv) in (c), clearly showing the associated mean spin excursion; (e) illustrates our proposed manipulation to freeze the squeezing direction along the $z$-axis with quasi-probability distribution. $\omega=(2 \pi) 2 \times 10^{4} \chi, \Omega_{0}=0.9057 \omega$ are used, starting with the initial state $\exp \left(i \Omega_{0} J_{y} / \omega\right)|j, j\rangle_{x}$ as in Fig. 3 .

were to treat the $\chi J_{z}^{2}$ and $\Omega(t) J_{y}$ terms as commuting with each other approximately at the large $\omega$ used.

Specifically for this case, our active rotation protocol involves turning off the modulated drive [Fig. 4(c)] when the squeezing parameter arrives at the optimal point [at $\Omega(t)=0]$, and performing a rapid state rotation to align squeezing direction to along the $z$-axis. The rotation operation consists of two steps: 1) a rotation along the $y$-axis by an angle $\Omega_{0} / \omega$ to align the mean spin direction in the equatorial plane, which results in the SS direction at $\pi / 4$ relative to the $z$-axis; and 2 ) a second rotation along the $-x$-axis by $\pi / 4$ to rotate the SS axis to along the $z$-axis, as illustrated in Fig. 4(e). The subsequent dynamics for the state is governed by the OAT Hamiltonian $H_{\mathrm{OAT}}$. The rotated squeezed state possesses a sharp distribution around $|j, 0\rangle$, whose squeezing parameter is frozen at the optimal point for a long time [Fig. 4(a)]. If we again take the same experimentally relevant value of $\chi \sim(2 \pi) 0.063 \mathrm{~Hz}$, the Heisenberg limit can still be reached even with $\omega$ and $\Omega_{0}$ reduced by five times to $\sim(2 \pi) 8 \mathrm{kHz}$.

In conclusion, we propose a scheme to freeze SS dynamics at theoretically determined optimal moments to achieve persistent (stationary) maximum SS. When applied to the two proposals [26, 27] for TACT SS at the Heisenberg limit, our scheme significantly relaxes stringent experimental requirements. The number of pulses required are drastically reduced in the repeated pulse proposal [26], and modulation frequency and amplitude in the modulated drive proposal [27] are also significantly reduced. The conditions for implementing our idea seem readily achievable in atomic SS laboratories. We believe its experimental realization will greatly advance the pursuit of ever increasing level of SS [12].

This work is supported by the MOST (Grant No. 2013CB922004 and No. 2014CB921403) of the National Key Basic Research Program of China, and by NSFC (No. 91121005, No. 11374176, and No. 11328404).

\section{Appendix A: The derivation of Eq. (4) in the main text}

The derivation of Eq. (4) in the main text is presented here starting with a system described by Hamiltonian

$$
H(t)=\chi J_{z}^{2}+\Omega_{0} \cos (\omega t+\varphi) J_{y} .
$$

A unitary transformation $U=\exp \left[-i \theta(t) J_{y}\right]$ with $\theta(t)=\int_{0}^{t} \Omega_{0} \cos \left(\omega t_{1}+\varphi\right) d t_{1}$, eliminates the timedependent coupling and reduces the Hamiltonian to

$$
\begin{aligned}
H & =U^{\dagger} H U-i U^{\dagger} \dot{U} \\
& =R_{y}(\varphi) \chi\left[J_{z} \cos \theta_{1}(t)-J_{x} \sin \theta_{1}(t)\right]^{2} R_{y}(-\varphi),
\end{aligned}
$$

where $R_{y}(\varphi)=\exp \left[-i \frac{\Omega_{0}}{\omega} \sin \varphi J_{y}\right]$ and $\theta_{1}(t)=$ $\frac{\Omega_{0}}{\omega} \sin (\omega t+\varphi)$. Making use of

$$
\begin{aligned}
& \cos [x \cos (\omega t)]=J_{0}(x)+2 \sum_{k>0}(-1)^{k} J_{2 k}(x) \cos (2 k \omega t), \\
& \sin [x \cos (\omega t)]=2 \sum_{k>0}(-1)^{k} J_{2 k-1}(x) \cos [(2 k-1) \omega t],
\end{aligned}
$$

and neglecting high-frequency oscillating terms in the high frequency approximation limit $\omega \gg \chi N$, we have

$$
\begin{aligned}
\cos ^{2} \theta_{1}(t) & \simeq\left[1+J_{0}\left(2 \Omega_{0} / \omega\right)\right] / 2, \\
\sin ^{2} \theta_{1}(t) & \simeq\left[1-J_{0}\left(2 \Omega_{0} / \omega\right)\right] / 2, \\
\sin \theta_{1}(t) \cos \theta_{1}(t) & \simeq 0 .
\end{aligned}
$$

Upon substituting Eq. (A2) into Eq. (A1), we arrive at

$$
H_{\mathrm{eff}}=\chi R_{y}(\varphi)\left[\alpha_{0} J_{z}^{2}+\left(1-\alpha_{0}\right) J_{x}^{2}\right] R_{y}(-\varphi)
$$

with $\alpha_{0}=\left[1+J_{0}\left(2 \Omega_{0} / \omega\right)\right] / 2$. 
[1] D. J. Wineland, J. J. Bollinger, W. M. Itano, F. L. Moore, and D. J. Heinzen, Phys. Rev. A 46, R6797 (1992); D. J. Wineland, J. J. Bollinger, W. M. Itano, and D. J. Heinzen, Phys. Rev. A 50, 67 (1994).

[2] A. Sørensen, L.-M. Duan, J. Cirac, and P. Zoller, Nature 409, 63 (2001).

[3] D. Leibfried, M. Barrett, T. Schaetz, J. Britton, J. Chiaverini, W. Itano, J. Jost, C. Langer, and D. Wineland, Science 304, 1476 (2004).

[4] C. Gross, T. Zibold, E. Nicklas, J. Esteve, and M. K. Oberthaler, Nature 464, 1165 (2010).

[5] M. F. Riedel, P. Böhi, Y. Li, T. W. Hänsch, A. Sinatra, and P. Treutlein, Nature 464, 1170 (2010).

[6] I. D. Leroux, M. H. Schleier-Smith, and V. Vuletić, Phys. Rev. Lett. 104, 073602 (2010).

[7] A. Louchet-Chauvet, J. Appel, J. J. Renema, D. Oblak, N. Kjaergaard, and E. S. Polzik, New Journal of Physics 12, 065032 (2010).

[8] V. Giovannetti, S. Lloyd, and L. Maccone, Nature Photonics 5, 222 (2011).

[9] C. Lee, J. Huang, H. Deng, H. Dai, and J. Xu, Frontiers of Physics 7, 109 (2012).

[10] R. J. Sewell, M. Koschorreck, M. Napolitano, B. Dubost, N. Behbood, and M. W. Mitchell, Phys. Rev. Lett. 109, 253605 (2012).

[11] E. Yukawa, G. J. Milburn, C. A. Holmes, M. Ueda, and K. Nemoto, Phys. Rev. A 90, 062132 (2014).

[12] J. G. Bohnet, K. C. Cox, M. A. Norcia, J. M. Weiner, Z. Chen, and J. K. Thompson, Nature Photonics 8, 731 (2014).

[13] C. Hamley, C. Gerving, T. Hoang, E. Bookjans, and M. Chapman, Nature Physics 8, 305 (2012).

[14] A. S. Sørensen and K. Mølmer, Phys. Rev. Lett. 86, 4431 (2001).

[15] J. K. Korbicz, J. I. Cirac, and M. Lewenstein, Phys. Rev. Lett. 95, 120502 (2005).

[16] J. K. Korbicz, O. Gühne, M. Lewenstein, H. Häffner, C. F. Roos, and R. Blatt, Phys. Rev. A 74, 052319 (2006).
[17] G. Tóth, C. Knapp, O. Gühne, and H. J. Briegel, Phys. Rev. A 79, 042334 (2009).

[18] L. Pezzé and A. Smerzi, Phys. Rev. Lett. 102, 100401 (2009).

[19] P. Hyllus, L. Pezzé, A. Smerzi, and G. Tóth, Phys. Rev. A 86, 012337 (2012).

[20] M. Kitagawa and M. Ueda, Phys. Rev. A 47, 5138 (1993).

[21] J. Ma, X. Wang, C. Sun, and F. Nori, Physics Reports 509, 89 (2011).

[22] C. Orzel, A. Tuchman, M. Fenselau, M. Yasuda, and M. Kasevich, Science 291, 2386 (2001).

[23] J. Esteve, C. Gross, A. Weller, S. Giovanazzi, and M. Oberthaler, Nature 455, 1216 (2008).

[24] T. Fernholz, H. Krauter, K. Jensen, J. F. Sherson, A. S. Sørensen, and E. S. Polzik, Phys. Rev. Lett. 101, 073601 (2008).

[25] R. Auccaise, A. G. Araujo-Ferreira, R. S. Sarthour, I. S. Oliveira, T. J. Bonagamba, and I. Roditi, Phys. Rev. Lett. 114, 043604 (2015).

[26] Y. C. Liu, Z. F. Xu, G. R. Jin, and L. You, Phys. Rev. Lett. 107, 013601 (2011).

[27] W. Huang, Y.-L. Zhang, C.-L. Zou, X.-B. Zou, and G.-C. Guo, Phys. Rev. A 91, 043642 (2015).

[28] K. Helmerson and L. You, Phys. Rev. Lett. 87, 170402 (2001).

[29] A. André and M. D. Lukin, Phys. Rev. A 65, 053819 (2002).

[30] I. Bouchoule and K. Mølmer, Phys. Rev. A 65, 041803 (2002).

[31] M. Zhang, K. Helmerson, and L. You, Phys. Rev. A 68, 043622 (2003).

[32] T. Opatrný, M. Koláŕ, and K. K. Das, Phys. Rev. A 91, 053612 (2015).

[33] J.-Y. Zhang, X.-F. Zhou, G.-C. Guo, and Z.-W. Zhou, Phys. Rev. A 90, 013604 (2014).

[34] G.-R. Jin and S. W. Kim, Phys. Rev. Lett. 99, 170405 (2007).

[35] J. H. Eberly, N. B. Narozhny, and J. J. SanchezMondragon, Phys. Rev. Lett. 44, 1323 (1980).

[36] C. K. Law, H. T. Ng, and P. T. Leung, Phys. Rev. A 63, 055601 (2001). 